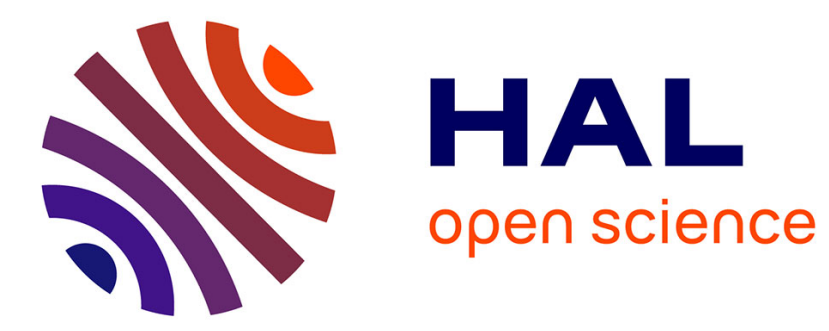

\title{
Virtual Chimera States for Delayed-Feedback Systems
} Laurent Larger, Bogdan Penkovskyi, Yuri Maistrenko

\section{To cite this version:}

Laurent Larger, Bogdan Penkovskyi, Yuri Maistrenko. Virtual Chimera States for Delayed-Feedback Systems. Physical Review Letters, 2013, 111, pp.054103. 10.1103/PhysRevLett.111.054103 . hal00878675

\section{HAL Id: hal-00878675 \\ https://hal.science/hal-00878675}

Submitted on 30 Oct 2013

HAL is a multi-disciplinary open access archive for the deposit and dissemination of scientific research documents, whether they are published or not. The documents may come from teaching and research institutions in France or abroad, or from public or private research centers.
L'archive ouverte pluridisciplinaire HAL, est destinée au dépôt et à la diffusion de documents scientifiques de niveau recherche, publiés ou non, émanant des établissements d'enseignement et de recherche français ou étrangers, des laboratoires publics ou privés. 


\title{
Virtual chimera states for delayed-feedback systems
}

\author{
Laurent Larger ${ }^{1}$, Bogdan Penkovsky ${ }^{1,2}$, and Yuri Maistrenko ${ }^{1,3}$ \\ ${ }^{1}$ FEMTO-ST / Optics Dept., UMR CNRS 6174, University of Franche-Comté, \\ 16 Route de Gray, 25030 Besançon Cedex, France \\ ${ }^{2}$ National University of Kyiv-Mohyla Academy, Skovorody Str. 2, 04655 Kyiv, Ukraine and \\ ${ }^{3}$ Institute of Mathematics and Center for Medical and Biotechnical Research, \\ NAS of Ukraine, Tereschenkivska Str. 3, 01601 Kyiv, Ukraine
}

(Dated: July 16, 2013)

\begin{abstract}
Time-delayed systems are found to display remarkable temporal patterns which dynamics split into regular and chaotic components repeating at the interval of a delay. This novel long-term behavior for delay dynamics results from strongly asymmetric nonlinear delayed feedback driving a highly damped harmonic oscillator dynamics. In the corresponding virtual space-time representation, the behavior is found to develop as a chimera-like state, a new paradigmatic object from the network theory characterized by co-existence of synchronous and incoherent oscillations. Numerous virtual chimera states are obtained and analyzed, through experiment, theory and simulations.
\end{abstract}

PACS numbers: 02.30.Ks, 05.10.-a, 05.45.Xt, 47.54.-r

Chimera states have been first highlighted theoretically at the edge of 2000 [1-4], and have soon after attracted great interest giving rise to a flurry of theoretical activity [5-9]. Recently only, first experimental evidence of chimera was obtained, independently in optical [10] and chemical [11] setups. The essence of the chimera phenomenon consists of hybrid-type dynamics when identical oscillators evolve into distinct groups, synchronous and incoherent, despite of homogeneous coupling. The conditions under which chimeras are expected, are usually concerned by a population of dynamical nodes characterized by extended non-local connections. Chimeras manifest then as coexisting clusters with different dynamical motions, e.g. phase-locked and chaotic, which can be interpreted as different parts of a whole body - the network - referring to different animal species, similarly to the creature of the Greek mythology. Motivated both by recent applied [12,13] and fundamental [14] results on delay dynamics, as well as by their space-time analogy $[15,16]$, particular conditions were numerically and experimentally explored, with the aim to reveal the existence of chimera-like motions in time-delayed systems.

Our particular system is an experimental realization of a modified Ikeda time-delayed equation [17]. It is modeled by a so-called bandpass nonlinear delay dynamics [18], and it gained recently sustained interest in the literature for many singular or unusual features compared to standard delay dynamics [19, 20], such as chaotic breathers [21], excitability and broadband chaos [22-25], stable one-delay periodic oscillations [14], to name only a few. In physical terms, the dynamics are described by an integro-differential time-delay equation which can be analyzed as corresponding to a strongly damped harmonic oscillator (with damping factor $m \gg 1$, and resonant angular frequency $\omega_{0}$ ), which is subject to a nonlinear delayed self-feedback term [26]. The generic equation of concern in this letter is as follows:

$$
\tau \frac{\mathrm{d} x}{\mathrm{~d} t}(t)+x(t)+\frac{1}{\theta} \int_{t_{0}}^{t} x(\xi) \mathrm{d} \xi=\beta \cdot f\left[x\left(t-\tau_{D}\right)\right]
$$
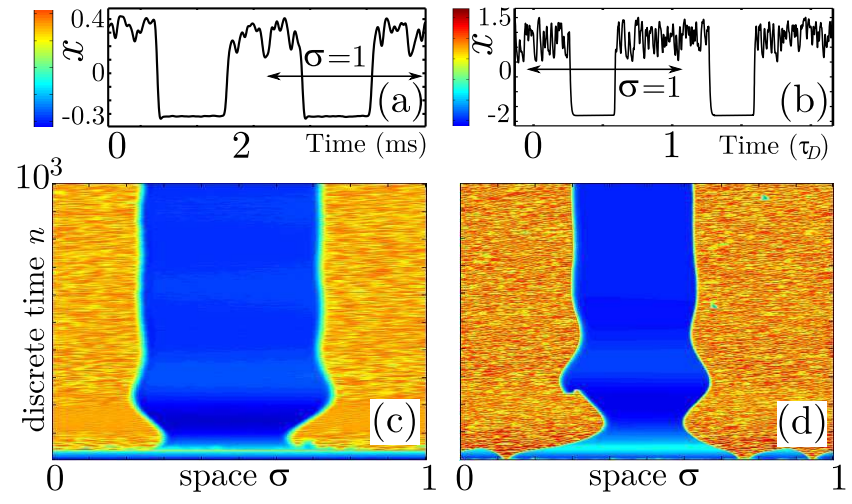

FIG. 1: (Color on line) Virtual chimera in delay dynamics. (a): Asymptotic temporal waveform of a chimera (arb. units in amplitude are encoded in color for the space-time plots). (b): Similar plots from numerical simulations of the experiment model, $\beta=0.6$ and $x_{0}=1$. (c,d): Virtual space-time representation of (a) and (b) respectively for 1000 steps, from the transient birth (lower) to the asymptotic state (upper). Left plots are experimental, right plots are numerics.

where $\tau \simeq\left(2 m \omega_{0}\right)^{-1}$ is the limited rate of change for the dynamical variable $x$ (fast time scale present in the Ikeda dynamics), $\theta \simeq 2 m / \omega_{0}$ is the characteristic time for the $x$-integral term (supplementary slow time scale compared to Ikeda dynamics). $\tau_{D}$ is the time delay, and $f[x]$ is the nonlinear transformation ruling the delayed self feedback term weighted by parameter $\beta$. Equation (1) can be rewritten in a more convenient $2 \mathrm{D}$ vectorial form, with a time scale $s=t / \tau_{D}$ normalized to the delay:

$$
\begin{aligned}
\varepsilon x^{\prime} & =-\delta y-x+\beta \cdot f[x(s-1)], \\
y^{\prime} & =x
\end{aligned}
$$

where $\varepsilon=\tau / \tau_{D}$ and $\delta=\tau_{D} / \theta$ are practically small quantities with $\varepsilon \cdot \delta \simeq(2 m)^{-2}$, since the delay time is both much larger than the characteristic response time, and much smaller than the integral time. 
The slow-fast system configuration modeled by Eq.(2) gives rise to many different types of asymptotic oscillatory behavior both in our experiments (see Fig.1(a)), as well as in the corresponding simulations (see Fig.1(b)). Following a standard procedure [15, 27], the delayedfeedback system given by Eq.(2) is analyzed in a virtual, spatially extended representation - by viewing every time moment $t$ as being $t=\tau_{c}(n+\sigma)$ with integer $n$, and real positive number $\sigma$ smaller than unity. This writing of the time allows to reveal the intrinsic multiple time scale features of a delay dynamical system within a space-time representation, as used in Fig.1(c,d): First a long time scale of the order of the delay is considered as a discrete time variable $n \in \mathbb{N}$; Second, a short time scale of the order of $\tau$ (or $\varepsilon$ in dimensionless units) is considered as a virtual continuous space variable $\sigma \in[0,1]$. The time $\tau_{c}$ is defined as $\tau_{D}(1+\gamma)$, with $\gamma$ being a small quantity of the order of $\varepsilon$. $\gamma$ is practically determined such that the virtual space-time plots actually feature pattern dynamics (as $n$ increases) that have a nearly stationary average position along the $\sigma$-horizontal axis.

A more detailed analysis on the scaling of $\gamma$ is given in $[16,27]$ based on the reduction of the delay dynamics to space-time dynamics. In particular, the rigorous equivalence between Ikeda time-delayed model and Ginzburg-Landau equation is obtained in the vicinity of a Hopf bifurcation, where parameter $\gamma$ corresponds to the velocity of the moving frame [27]. With the help of this construction, purely temporal phenomena in delayed systems admit a transparent visualization in terms of spatio-temporal patterns. Applying it to a recently observed and analyzed new one-delay periodic square wave solution of Eq.(2) [14], one can interpret this solution as two clusters of oscillators continuously distributed in the $\sigma$-space: The clusters are expected to correspond to alternated plateaus with constant amplitude. As time $n$ is iterated, these two clusters are maintained in the virtual space, since the global motion is indeed found to be stable, in the presence of the integral term [28].

In this Letter, the virtual cluster concept is pushed even further, showing much more differentiated (incongruous, as expressed in [2]) motions: one or several clusters manifest themselves as quiet plateaus (as in [14]), whereas other ones exhibit chaotic fluctuations. This coexisting incongruous behavior becomes possible due to strong asymmetry of the nonlinear transformation $f[x]$. The usual shape involved in the standard Ikeda delay dynamics is indeed symmetric, as in [14]. As shown in Fig.1(c,d), a virtual chimera state arises in the space-time representation of Eq.(2). It is worth noticing that these exotic spatial patterns are maintained over thousands of time steps $n$, although its cluster frontiers, as reported later, exhibit small chaotic wandering (cf. [6]).

Our results are supported by experiments, modeling, and simulations. The physical experiment corresponds to a bandpass FM (Frequency Modulation) delay oscillators which setup is depicted in Fig.2(a) and which modeling can be derived as corresponding to Eq.(2). The
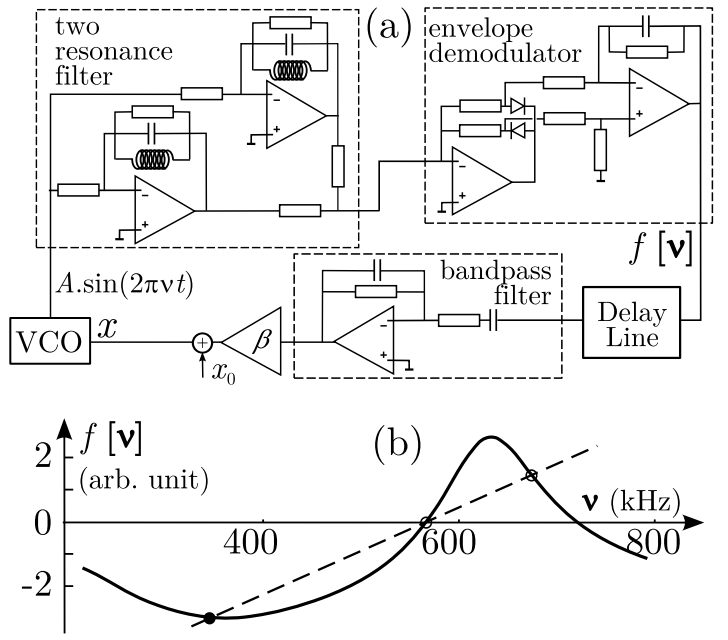

FIG. 2: Experiment. (a) Setup of the electronic FM nonlinear delay oscillator. (b) Physical non linearity, as a function of the VCO output frequency $\nu$, with the first bisector (dashed line) revealing the fixed points of the associated map.

setup is directly inspired by the FM chaotic oscillator initially reported in $[18,30]$. Its modeling in the form of Eq.(1) can be analyzed as follows. The left hand side of the equation appears as the linear dynamics of a strongly damped harmonic oscillator, from a physics interpretation; From a more signal theory viewpoint, it corresponds to a broadband bandpass second order filter. The filter is characterized in the Fourier domain by a low cut-off $f_{l}=(2 \pi \theta)^{-1}$ (responsible for the integral term and the slow motion), and a high cut-off $f_{h}=(2 \pi \tau)^{-1}$ (responsible for the differential term, the fast motion, see [26] for modeling details). The right hand side consists of the nonlinear delayed contribution. The advantage of our FM delay dynamics consists in the possibility for an easy and accurate design of the nonlinear function shape, as already used in [30] in the context of sub-critical Hopf bifurcation in Ikeda-like dynamics. In order to observe virtual chimera, a key issue is to design a nonlinear transformation with two strongly asymmetric successive extrema: A broad minimum is connecting a sharp maximum, via a positive (feedback) slope, as illustrated in Fig.2(b). This asymmetry feature can be easily designed with the FM delay dynamics, in which the nonlinear function is reduced to an amplitudefiltering profile in the frequency range of the FM carrier signal. As depicted in Fig.2(a), the setup involves a VCO (voltage controlled oscillator), which output frequency is proportional to the input voltage $x(t)$ $\left(\nu=\nu_{0}+S_{\nu} x\right.$ varying in $\left.[150 ; 800] \mathrm{kHz}\right)$. The FM signal at the $\mathrm{VCO}$ output is thus filtered by a dual resonance filter (resonances at $\nu_{1}=210 \mathrm{kHz}$ and $\nu_{2}=600 \mathrm{kHz}$ ). A dual-wave rectifier followed by a low-pass filter (LPF), allows for the amplitude demodulation of the filtered FM signal. The corresponding transformation from VCO-input to LPF-output, performs the desired 
function $f[x]$ in Eq.(1), as it can be theoretically derived:

$$
f[\nu]=\left|\frac{g_{12} i \frac{\nu}{\nu_{1}}}{1-\left(\frac{\nu}{\nu_{1}}\right)^{2}+\frac{i}{Q_{1}} \frac{\nu}{\nu_{1}}}+\frac{i \frac{\nu}{\nu_{2}}}{1-\left(\frac{\nu}{\nu_{2}}\right)^{2}+\frac{i}{Q_{2}} \frac{\nu}{\nu_{2}}}\right| .
$$

The resulting experimental nonlinear function is plotted in Fig.2(b), together with the first bisector (dashed) line highlighting the fixed points of the associated map $x_{n}=f\left[x_{n-1}\right]$. A central unstable fixed point is revealed, surrounded by two other ones: one potentially stable around a broad minimum, and another potentially unstable around a sharp maximum.

The nonlinear transformation is then delayed in time by an electronic delay line based on a FIFO memory (first in first out, 4096 depth, $3.2 \mathrm{MHz}$ sampling rate), providing a delay $\tau_{D}=2.53 \mathrm{~ms}$. The delayed signal is then filtered by a second order bandpass filter having low and high cut-off frequencies of $f_{l}=1 \mathrm{~Hz}$ and $f_{h}=12.5 \mathrm{kHz}$ respectively. This filter rules the integro-differential dynamics for $x(t)$ in the left hand side of Eq.(1). According to this modeling, the normalized parameters characterizing the slow and fast motion in Eq.(2) are $\varepsilon \simeq 5.0 \times 10^{-3}$ and $\delta \simeq 1.6 \times 10^{-2}$ respectively (leading to a corresponding damping $m \simeq 56$ ). Before applying $x(t)$ to the VCO, amplification and offset can be adjusted, thus controlling the overall feedback gain $\beta$ and an offset $x_{0}$. These two important parameters are setting the weight for $f[x]$, and the horizontal position of the graph in Fig.2(b) relatively to the bisector line.

Numerical simulations were conducted using the dynamics in Eq.(2) and the nonlinear function in Eq.(3) by explicit Runge-Kutta methods for delay differential equations with random initial conditions over a delay interval. Various parameter conditions for $\beta$ and $x_{0}$ were scanned, with fixed normalized parameters for the nonlinear function shape $\left(\left[Q_{1} ; \nu_{1}\right]=[6 ; 6],\left[Q_{2} ; \nu_{2}\right]=[2 ; 1.8], g_{12}=1\right.$, $x \in[3.4 ; 7.4])$. These conditions led to very good qualitative agreement between experiments and numerics.

Figures 1 and 3 represent typical examples of the virtual chimeras and other dynamical regimes that have been observed with our model and experiment. Among them, the particular space-time plots from Fig.3(a-d) have been called multiple-headed chimera, following further the Greek mythology analogy. They were again observed both from experiments and numerics, as coexisting with the single-headed solution in Fig.1. The different states are obtained by fixing the system parameters and by consecutively resetting the initial conditions (corresponding to small noise amplitude of $x(s)$ $s \in[-1,0])$. As in Fig.1(c,d), the space-time plots show that each chimera-like pattern stabilizes after a transient motion, lasting then over several thousands units of $n$. Shape of the vitual chimera state is determined by the relative spatial length of the regular and chaotic intervals in the space-time representation. The single-headed chimera has the only one interval of chaoticity which
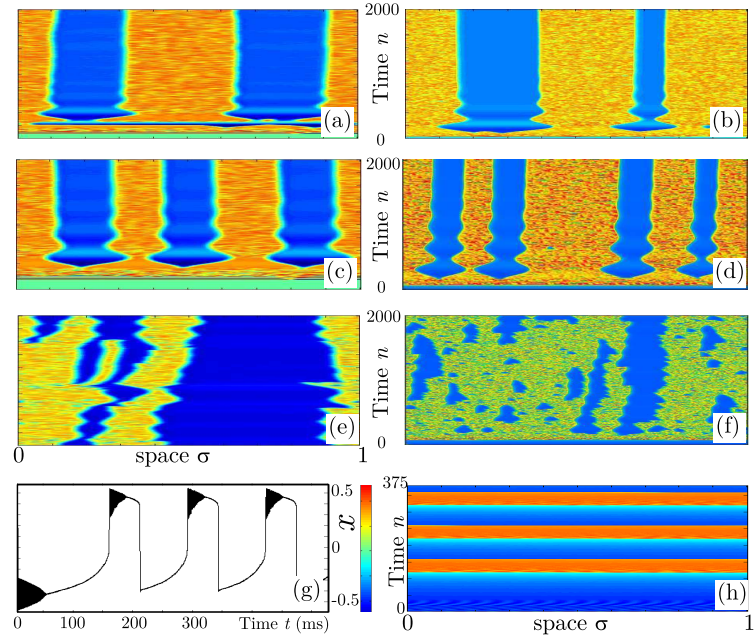

FIG. 3: (Color on line) Diversity of motions. Two-headed chimera, (a): Exp. (b): Num. Three-headed ((c): Exp.) and four-headed ((d): Num. with $\beta=0.6$ and $\left.x_{0}=1\right)$ chimera. Turbulent motion, (e): Exp.; (f): Num. (g,h): Chaotic experimental breathers; Time trace in $(\mathrm{g})$ with colored amplitude scaling for the space-time plot in (h).

asymptotic length does not depend on the initial conditions. The two-headed chimera (Fig.3(a,b)) contains two different chaotic intervals. However, their accumulated length is found to be invariant in the sense that it does not depend on the initial conditions neither, but it does depend on the system parameters. Even more exotic, three- and four-headed virtual chimera states (Fig.3(c,d)) can be observed, although their appearance is essentially less probable when starting the dynamics from randomly chosen initial conditions. This occurrence probability has been found to be gain-dependent: For low $\beta$-values, essentially single-headed chimera are obtained, whereas multiple-headed chimeras become more probable for higher $\beta$-values. We believe that there should also exist multi-headed virtual chimera states with order higher than 4. However their probability to occur seems to be so small that one needs specially prepared initial conditions to obtain them.

If $\beta$ is further increased, the virtual chimeras disappear, giving rise to space-time turbulence as illustrated in Fig.3(e,f) from experiments and numerics respectively. Interestingly, other known solutions have been found to co-exist with chimeras (Fig.3(g,h)), the so-called chaotic breathers [21], which occurrence essentially depends on initial conditions. In the space-time representation these are long waves, where non-constant regular and growing chaotic behaviors are alternated, resembling neuronal chaotic bursting [31].

Alternatively, decreasing the gain below the single headed chimera threshold, results eventually in the asymmetric square-wave dynamics described previously in [14]. A crucial role for the virtual chimera existence and stability is also played by the magnitude of the integral 
term in Eq.(2) (parameter $\delta=\tau_{D} / \theta$ ). Indeed, as $\delta$ decreases, the probability to obtain chimera-like behavior, both in experiment and simulations drops down, which means apparently that its basin of attraction shrinks. The virtual chimera state loses stability at some value $\delta_{\text {bif }}>0$ (as we assume, in an inverse subcritical Hopf bifurcation), such that for $\delta<\delta_{b i f}$ the sytem trajectories start to approach whether the lower equilibrium, or the upper chaotic attractor given by the nonlinear transformation $f[x]$ (shown in Fig.2(b)). Interestingly, as $\delta$ approaches the bifurcation value $\delta_{b i f}$, the transient times grow so fast that accurate numerical identification of the bifurcation value becomes nontrivial and time consuming. Here are a few identified bifurcation values: $\delta_{b i f}=3.10^{-4} ; 10^{-4} ; 3.10^{-5}$ for $\varepsilon=0.03 ; 0.02$; and 0.01 respectively (other parameters are as in Fig.1(d)). We were not able to observe this bifuration in experiment because of the limited experimental $\delta$-tuning range (difficulties to set small values).
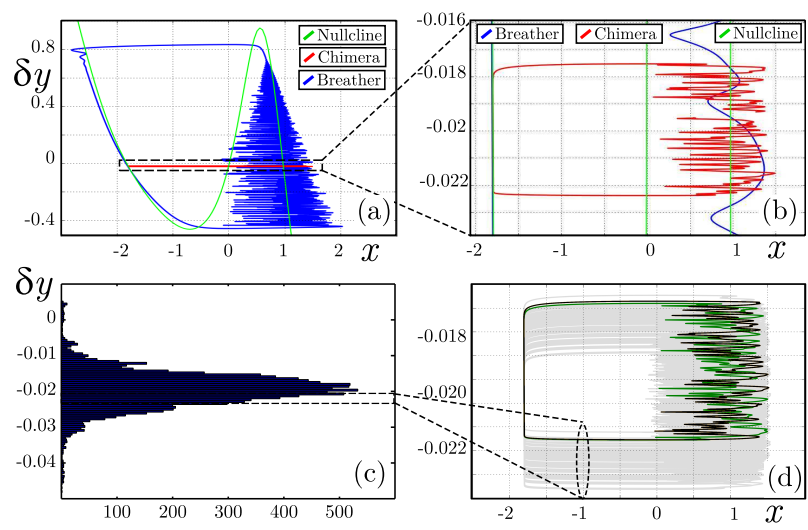

FIG. 4: (Color on line) Nullcline numerical analysis of the chimera, $\beta=0.6, x_{0}=1$. (a): Chaotic breathers (blue), as in Fig.3(c,d), and chimera (red) along the nullcline (green). (b): Zoom-in of (a) close to $y=0$. (c): Histogram for $\delta y$-values at the condition $x=-1.0$ (see circle in (d)), over 10,000 delays. (d): Chimera wandering in the $(x, \delta y)$-plane, for two (black and green) and 100 (light gray) successive delays.

In the two-dimensional phase space given by Eq.(2) the chimera-like dynamics are localized in a narrow stripe around the $y=0$ axis related to the nullcline $\delta y=$ $-x+f[x]$ (see Fig.4). The trajectory is here revealed as successive fast jumps from the left to the right and back, following the slow nullcline branches, but only over the short time interval given by a fraction of the time delay $\tau_{D}$ (Fig.4(b,d)). The chimera-like behavior arises due to different dynamics on the left and on the right: the behavior is monotonic when sliding down the left nullcline branch (regular part of the chimera), and chaotic on the right (irregular chimera part). The behavior is different from those of the chaotic breathers (blue trace in Fig.(4): The latter indeed follows the entire nullcline contour passing close to its two extrema. As it is also illustrated in Fig.4(c,d), the two-dimensional chimera contour is not periodically repeating at each iteration of $n$, but it is slowly moving randomly back and forth along the $\delta y$-axis. This causes the property of chaotic wandering of the virtual chimera state, which is typical for chimeras in oscillatory networks $[6,29]$. However, in our case, the wandering does not lead to chimera collapse as it usually takes place in oscillatory networks [29, 33].

The analogy of our virtual chimera state with the original chimera state introduced by Kuramoto and Strogatz, can be drawn as follows, using the already recalled spatio-temporal interpretation of delay dynamics. The integro-differential dynamics in Eq.(1) can be reformulated as a "spatially global" convolution equation ruling the discrete time update of oscillator $x_{n}(s)$, from time step $(n-1)$ to time step $n$ :

$$
x_{n}(s)=\int_{0}^{s} h(\xi) f\left[x_{n-1}(s-\xi)\right] \mathrm{d} \xi,
$$

where $h(s)$ is the (causal) impulse response of the linear bandpass filter described by the left hand side of Eq.(1). In the previous convolution product, one clearly sees that the updated oscillator at time $n$ for the position $s$ depends non linearly, through the function $f$, on itself and its neighbors at the previous time $(n-1)$. The weighting function defining the coupling radius with adjacent neighbors at position $\xi$ from $s$, appears as $h(\xi)$. In the case considered, the coupling radius in $s$ can be evaluated as the zero crossing of $h(\xi)$, which is approximately $\left(m \omega_{0}\right)^{-1} \ln (2 m)=\tau \ln (\theta / \tau)=-\tau_{D} \varepsilon \ln (\varepsilon \delta)$. At fixed $\varepsilon$, the coupling radius would decrease as $(-\ln \delta)$.

In conclusion, we have identified a novel mechanism for the structure emergence in time-delayed systems by exploiting the correspondence with one-dimensional, spatially extended system. It consists in the appearance of multistable virtual chimera states of different modality observed both in experiment and simulations, with very good qualitative agreement. The reported results are found robust with respect to system parameters. This indicates a common, probably universal phenomenon in delayed feedback systems of very different nature.

We thank Martin Hasler, Eckehard Schöll, and Marc Timme for illuminating discussions. This work was supported by the European project PHOCUS (FP7 grant 240763), and by the Labex ACTION program (contract ANR-11-LABX-01-01). Y.M. and B.P. acknowledge the support of the Region Franche-Comté for the funding of their stay at FEMTO-ST.
[1] Y. Kuramoto and D. Battogtokh, Nonlinear phenomena in complex systems 5, 380 (2002).
[2] D. M. Abrams and S. H. Strogatz, Phys. Rev. Lett. 93, 
174102 (2004).

[3] S. I. Shima and Y. Kuramoto, Phys. Rev. E 69, 036213 (2004).

[4] H. Sakaguchi, Phys. Rev. E 73, 031907 (2006).

[5] O. E. Omel'chenko, Y. L. Maistrenko, and P. A. Tass, Phys. Rev. Lett. 100, 044105 (2008); D. Abrams, R. Mirollo, S. Strogatz, and D. D. Wiley, Phys. Rev. Lett. 101, 084103 (2008); G. C. Sethia, A. Sen, and F. M. Atay, Phys. Rev. Lett. 100, 144102 (2008); C. Laing, Physica D 238 (2009); E. A. Martens, C. R. Laing, and S. H. Strogatz, Phys. Rev. Lett. 104, 044101 (2010); G. Bordyugov, A. Pikovsky, and M. Rosenblum, Phys. Rev. E 82, 035205 (2010); M. Wolfrum, O. Omelchenko, and S. Yanchuk, Chaos 21, 013112 (2011); I. Omel'chenko, Y. L. Maistrenko, P. Hövel, and E. Schöll, Phys. Rev. Lett. 106, 234102 (2011); W. Lee, J. Restrepo, E. Ott, and T. Antonsen, Chaos 21, 023122 (2011); O. E. Omel'chenko, M. Wolfrum, S. Yanchuk, Y. L. Maistrenko, and O. Sudakov, Phys. Rev. E 85, 036210 (2012).

[6] O. E. Omel'chenko, M. Wolfrum, and Y. L. Maistrenko, Phys. Rev. E 81, 065201(R) (2010).

[7] C. Laing, K. Rajendran, and I. Kevrekidis, Chaos 22, $013132(2012)$

[8] Y. Zhu, Y. Li, M. Zhang, and J. Yang, Europhys. Lett. 97, 10009 (2012).

[9] M. J. Panaggio and D. M. Abrams, Phys. Rev. Lett. 110, 094102 (2013).

[10] A. M. Hagerstrom, T. E. Murphy, R. Roy, P. Hövel, I. Omelchenko, and E. Schöll, Nature Physics (London) 8, 658 (2012).

[11] M. Tinsley, S. Nkomo, and K. Showalter, Nature Physics (London) 8, 662 (2012).

[12] L. Larger, M. C. Soriano, D. Brunner, L. Appeltant, J. M. Gutierrez, L. Pesquera, C. R. Mirasso, and I. Fischer, Opt. Express 20, 3241 (2012).

[13] R. Martinenghi, S. Rybalko, M. Jacquot, Y. K. Chembo, and L. Larger, Phys. Rev. Lett. 108, 244101 (2012).

[14] L. Weicker, T. Erneux, O. d'Huys, J. Danckaert, M. Jacquot, Y. Chembo, and L. Larger, Phys. Rev. E 86, 055201(R) (2012).

[15] F. T. Arecchi, G. Giacomelli, A. Lapucci, and R. Meucci, Phys. Rev. A 45, R4225 (1993).

[16] G. Giacomelli, F. Marino, M. A. Zaks, and S. Yanchuk, Europhys. Lett. 99, 58005 (2012).

[17] K. Ikeda, Optics Commun. 30, 257 (1979).

[18] V. S. Udaltsov, L. Larger, Goedgebuer, M. W. Lee, E. Genin, and W. T. Rhodes, IEEE Trans. Circuits Syst. I 49, 1006 (2002).

[19] M. Peil, M. Jacquot, Y. K. Chembo, L. Larger, and T. Erneux, Phys. Rev. E 79, 026208 (2009).
[20] T. E. Murphy, A. B. Cohen, B. Ravoori, K. R. B. Schmitt, A. V. Setty, F. Sorrentino, C. R. S. Williams, E. Ott, and R. Roy, Phil. Trans. R. Soc. A 368, 343 (2010).

[21] Y. C. Kouomou, P. Colet, L. Larger, and N. Gastaud, Phys. Rev. Lett. 95, 203903 (2005).

[22] A. Argyris, D. Syvridis, L. Larger, V. Annovazzi-Lodi, P. Colet, I. Fischer, J. Garcia-Ojalvo, C. R. Mirasso, L. Pesquera, and A. K. Shore, Nature (London) 438, 343 (2005).

[23] K. E. Callan, L. Illing, Z. Gao, D. J. Gauthier, and E. Schöll, Phys. Rev. Lett. 104, 113901 (2010).

[24] L. Larger and J. M. Dudley, Nature 465, 41 (2010).

[25] D. D.Rosin, K. Callan, D. Gauthier, and E. Schoell, Europhys. Lett. 96, 34001 (2011).

[26] L. Larger, Phil. Trans. Roy. Soc. A "accepted for publication" (2013).

[27] G. Giacomelli and A. Politi, Phys. Rev. Lett. 76, 2686 (1996).

[28] These "a priori" simple solutions are known to be unstable in conventional (without the integral term) nonlinear delay dynamics. For example, this issue applies to the well known Ikeda time-delay equation with sinusoidal, or even more general nonlinearity (e.g. MackeyGlass dynamics). The instability of the solutions was proved mathematically to be valid for any small $\varepsilon>0$ [32]. The evidence of vanishing patterns in the spatiotemporal representation, including nucleation, propagation, and annihilation of fronts, and leading eventually to a single phase, were recently reported from laser with delayed opto-electronic feedback [16].

[29] As shown in [33], chimeras are transient states, transient time of them grows exponentially along with system size. Our model is infinite-dimensional in experiment and higher-dimensional, up to $N=10000$, in numerical simulations. Both experiment and numerics have shown robust virtual chimera patterns of several thousands of iterations. Unstable chimera states were also found, but in parameter conditions $\delta \ll 1$ that are asymptotically corresponding to the results of Ref. [16, 32].

[30] L. Larger, J.-P. Goedgebuer, and T. Erneux, Phys. Rev. E 69, 036210 (2004).

[31] T. R. Chay and J. Rinzel, Biophys. J. 47, 357 (1985).

[32] A. Sharkovsky, Y. Maistrenko, and E. Romanenko, Difference Equations and their Applications (Kluwer Acad. Publ. (Naukova Dumka, Kiev, in Russian, 1986), 1993), chap. 3.

[33] M. Wolfrum and O. Omel'chenko, Phys. Rev. E 84, 015201 (2011). 